\title{
Answer to the Letter to the Editor concerning "Pedicle screw fixation of thoracolumbar fractures: conventional short segment versus short segment with intermediate screws at the fracture level-a systematic review and meta-analysis" by C. Kapoen, et al. [Eur Spine J; 2020; 29(10):2491-2504]
}

\author{
Carolijn Kapoen $^{1} \cdot$ Yang Liu ${ }^{1}$. Frank W. Bloemers ${ }^{1} \cdot$ Jaap Deunk ${ }^{1}$
}

Received: 16 July 2021 / Accepted: 18 July 2021 / Published online: 3 August 2021

๑) The Author(s), under exclusive licence to Springer-Verlag GmbH Germany, part of Springer Nature 2021

First of all, we would like to thank the authors of the Letter to the Editor for taking the time to respond to our systematic review and putting some critical notes to the results.

Let's start to state that we fully agree with the authors that, given the quality of the present literature, the results of our review and meta-analysis should be interpreted with some caution and conclusions are not definite. We believe this was sufficiently acknowledged in our discussion. Moreover, we explicitly stated in our conclusion that the overall quality of evidence was moderate to low and that more studies on this topic are needed.

In response to the critical notes on our search methods (databases and search terms used), we agree that perhaps some choices do not conform to the ideal methodological standards. However, we believe that the most important databases (in which all well-known and less-known journals on spinal topics are included) were used in this systematic review. Moreover, all included articles in this review were checked for their references for additional articles and even Chinese language manuscripts were included (and translated) for this review. We were therefore able to include 27 studies in our review, significantly more than in previous published reviews on this topic. We doubt whether there is any relevant study on this topic that is not included in our review. But even if there is one, the question always remains what the scientific contribution is of such a study and whether this would have had any influence on the conclusions of our review. Although some choices could have

Jaap Deunk

j.deunk@amsterdamumc.nl

1 Department of Trauma Surgery, Amsterdam UMC, VU University Medical Center, 1081 HV Amsterdam, The Netherlands been different ideally, we still think this is the best and most comprehensive review on this topic up to date.

We fully agree on the issue of heterogeneity of the studies in this review. The methodological heterogeneity (heterogeneous populations, different fracture levels, different indications, etc.) was already discussed in our discussion, and we made a short notification of the statistical heterogeneity as well. The heterogeneity in the results was not explicitly analyzed nor discussed since, according to our opinion, this is of less importance if the studies are already found to be methodologically heterogeneous. The quality of the evidence is explicitly valuated to 'moderate' or 'low', which should give the reader a good impression of the evidence and how definite the conclusions are. But perhaps this should have been pronounced even more.

We decided to address the outcomes in this review because these were the outcomes used in the included studies and all considered clinically relevant. Since not all studies used the same but alternative outcomes, reducing the number of outcomes would have resulted in excluding some of the studies and their important data. We believe that reducing the number of outcomes would have introduced a selective reporting instead of improving the quality, but perhaps at the expense of the focus.

So overall, we agree with the authors of the Letter to the Editor that high-quality evidence on this topic is still lacking and therefore results should be interpreted with caution and conclusions are not definite. Hopefully, this review will inspire readers to initiate higher quality studies on this topic!

Publisher's Note Springer Nature remains neutral with regard to jurisdictional claims in published maps and institutional affiliations. 